Carnets de géographes

GÉOGRAPHES

\title{
La gestion des risques industriels : l'exemple de l'ile Mandji au Gabon
}

\section{Gisèle Lila Miyagou}

\section{(2) OpenEdition}

1 Journals

Édition électronique

URL : http://journals.openedition.org/cdg/940

DOI : $10.4000 / c d g .940$

ISSN : 2107-7266

Éditeur

UMR 245 - CESSMA

Référence électronique

Gisèle Lila Miyagou, « La gestion des risques industriels : l'exemple de l'ile Mandji au Gabon », Carnets de géographes [En ligne], 6 | 2013, mis en ligne le 01 septembre 2013, consulté le 23 septembre 2020. URL : http://journals.openedition.org/cdg/940 ; DOI : https://doi.org/10.4000/cdg.940

Ce document a été généré automatiquement le 23 septembre 2020.

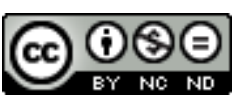

La revue Carnets de géographes est mise à disposition selon les termes de la Licence Creative Commons Attribution - Pas d'Utilisation Commerciale - Pas de Modification 4.0 International. 


\title{
La gestion des risques industriels : l'exemple de l'ile Mandji au Gabon
}

\author{
Gisèle Lila Miyagou
}

Cette thèse constitue une recherche qui fait écho à une question d'importance, celle de la prise en compte des risques industriels sur l'un des plus grands sites pétrolier et industriel du Gabon : l'île Mandji. Cette île abrite Port-Gentil, capitale économique du Gabon peuplée de plus de 100000 habitants et héberge une diversité d'industries situées le long du littoral. L'industrialisation et l'urbanisation sur l'île se sont développées de façon parallèle comme le montre l'évolution de la situation de 1960 à 2010 (Figure 1). 
Figure 1 : Evolution de l'industrialisation et de l'urbanisation de 1960 à nos jours

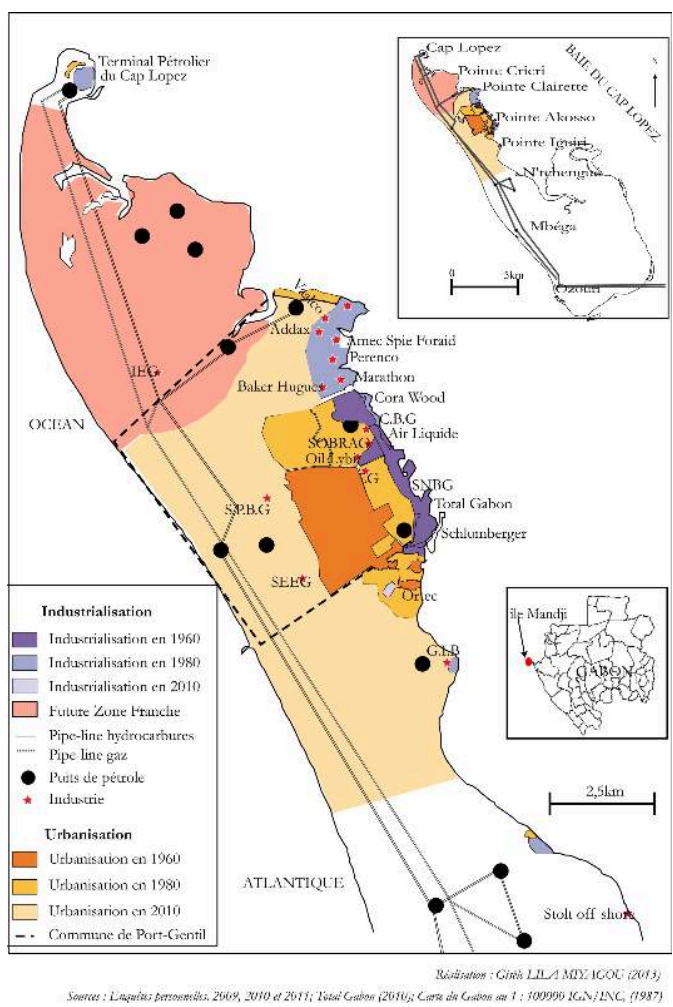

2 Le choix de ce site s'explique entre autres raisons, par le fait que c'est un territoire aux données lacunaires notamment sur les risques industriels qui est un sujet inédit sur l'île Mandji. Cette problématique renvoie à deux enjeux primordiaux: celui du développement durable et celui de la sécurité environnementale, qui sont aujourd'hui au centre des débats au Gabon et sur la scène internationale.

3 L'objectif de cette recherche est d'identifier les facteurs de risques industriels, d'évaluer la vulnérabilité des populations, des biens et des écosystèmes exposés, d'analyser le lien industrie-aménagement et de proposer des outils susceptibles de réduire la vulnérabilité du territoire pour un développement durable.

4 Cette étude s'inscrit dans un contexte économique et politique particulièrement délicat, lié à la présence des multinationales, où l'accès aux informations reste extrêmement difficile. Cette situation m'a incitée à mettre en place une démarche exploratoire associant différentes entrées méthodologiques pour combler l'insuffisance des données sur le risque industriel.

5 Ma première démarche consiste à mettre l'accent sur les fondements théoriques de la thèse et sur la présentation empirique de mon terrain d'étude en partant de deux concepts clés : vulnérabilité et risque industriel.

6 La deuxième approche propose différentes analyses sur la représentation des risques industriels. Une spatialisation des enjeux et des aléas, fondée sur la construction d'une base de données sur le Risque Industriel Ile Mandji (RIIM) a été développée. L'idée est de présenter le rôle structurant de la cartographie dans la gestion des risques pour rendre visible la vulnérabilité de ce territoire et affirmer l'utilité de cet outil dans la gestion durable de l'île. L'étude de la représentation passe aussi par une analyse fondée sur des entretiens et des enquêtes. L'ensemble de ces données quantitatives et qualitatives m'a permis de dégager un certain nombre d'éléments caractérisant la 
culture du risque des différents acteurs (habitants, industriels et pouvoirs publics) et d'appréhender leur rôle respectif.

7 La troisième approche concerne les instruments de prise en compte du risque industriel dans l'aménagement durable. Elle fait un état des lieux de l'encadrement juridique des risques industriels, de l'urbanisme et précise leurs limites. Il existe donc deux dispositifs normatifs qui encadrent ces risques aujourd'hui au Gabon. Le dispositif réglementaire dont le texte fondamental est la loi du 26 août 1993 relative à l'amélioration et à la protection de l'environnement dit «code de l'environnement ». Ce code n'est pas suffisamment appliqué. Il existe un dispositif contractuel qui est antérieur au code de l'environnement qui remplit finalement le rôle d'encadrement juridique dont les critères d'application sont définis par les acteurs économiques privés.

8 La thèse met aussi l'accent sur le développement de la Responsabilité Sociale des Entreprise (RSE) en particulier des multinationales considérées comme les véritables acteurs de gestion de risques industriels tout comme sur les nouvelles pratiques d'aménagement par les pouvoirs publics.

Enfin, trois scenarii d'aménagement de l'île Mandji fondés respectivement sur le degré de vulnérabilité, la mise en place de la Zone Franche de l'île Mandji et le déplacement hypothétique du terminal pétrolier du Cap Lopez dans la prévention des quartiers spontanés traversés par les pipe-lines, ont été mis en place.

Les facteurs de risques industriels sur l'île Mandji sont multiples, notamment les industries, scieries (risques sites), le réseau de pipe-lines hydrocarbures, de pipe-lines eaux ou du réseau routier par lequel transitent les matières dangereuses (risques transports) et les bourbiers, les industries et pipe-lines en friche (risques friches). La combinaison de ces risques peut complexifier leur gestion et participer à la vulnérabilité de l'île. A cela s'ajoute d'autres facteurs de vulnérabilité. Par exemple l'urbanisation spontanée qui est liée au droit coutumier parfois en contradiction avec le droit positif de l'urbanisme. Cette vulnérabilité est également accentuée par la topographie, les inondations et la localisation des industries dans la zone littorale. Enfin la vulnérabilité sur l'île est encore accentuée par l'absence de culture de risque des populations. Un dernier élément a contribué à ne pas réduire la vulnérabilité : l'insuffisance d'un droit réglementaire et son application par les pouvoir publics. Cependant, il existe une gestion de risque industriel qui repose sur un certain nombre d'acteurs publics et privés. Cette gestion n'est peut-être pas encore optimale car elle repose pour partie sur des accords volontaires mais elle pourrait-être un levier pour une meilleure prise en compte des risques industriels par l'Etat.

\author{
Discipline \\ Géographie et aménagement \\ Directeur \\ Helga Jane SCARWELL, Professeure \\ Université \\ Lille 1 Sciences et Technologies
}

Membres du jury de thèse, soutenue le 19 juin 2013

Richard LAGANIER, Professeur, Université Paris-Diderot 
Christophe BEAURAIN, Professeur, Université de Limoges

Jean Bernard MOMBO, Professeur, Université Omar Bongo de Libreville

Christophe GIBOUT, Professeur, Université du Littoral Côte d'Opales de Dunkerque

David GILBERT, Directeur de Recherche à l'Institut de Recherche et du

Développement

Courriel de l'auteur

miyagou@yahoo.fr

INDEX

Thèmes : Carnets de soutenances 